\title{
N. H. HEINEN, Proverbes égyptiens relatifs aux poissons
}

et aux oiseaux

(traduction française Ch. Vial). Le Caire, Institut français d'archéologie orientale, 2007 « Bibliothèque générale, 30 », 367 p.

\section{Katia Zakharia}

\section{(2) OpenEdition}

12 Journals

Édition électronique

URL : http://journals.openedition.org/beo/104

DOI : 10.4000/beo.104

ISBN : 978-2-35159-316-5

ISSN : 2077-4079

Éditeur

Presses de l'Institut français du Proche-Orient

Édition imprimée

Date de publication : 1 septembre 2009

Pagination : 425-428

ISBN : 978-2-35159-143-7

ISSN : 0253-1623

\section{Référence électronique}

Katia Zakharia, « N. H. HEInEN, Proverbes égyptiens relatifs aux poissons et aux oiseaux », Bulletin d'études orientales [En ligne], Tome LVIII I Septembre 2009, mis en ligne le 29 septembre 2009, consulté le 22 septembre 2020. URL : http://journals.openedition.org/beo/104 ; DOI : https://doi.org/10.4000/beo. 104 
PROVERBES ÉGYPTIENS RELATIFS AUX

POISSONS ET AUX OISEAUX

(traduction française $\mathrm{Ch}$. Vial).

\section{N. H. HEINEN}

Le Caire, Institut français d'archéologie orientale, 2007 « Bibliothèque générale, 30 », 367 p. Texte arabe: p. 1-124; p. 125-166 index des termes arabes cités dans les proverbes. Texte français : p. 1-165; p. 167-177 : annexe 1 (diverses versions des proverbes) ; p. 179-194: tableau des concordances.

Il s'agit d'un ouvrage agréable à lire, dans lequel sont consignés 369 proverbes ayant en commun la thématique annoncée dans le titre à savoir les poissons ou les oiseaux. Le corpus a été rassemblé, pour une petite part, lors d'une "enquête de terrain », spontanément entreprise par l'auteur durant un séjour d'apprentissage professionnel « à Mațariyya, qui domine le lac Manzala » (p. 2). Il a été complété par des relevés effectués dans neuf sources manuscrites ou imprimées, s'étendant du $\mathrm{Ix}^{\mathrm{e}}$ au $\mathrm{xx}^{\mathrm{e}}$ siècles et rapidement présentées dans l'introduction.

Les proverbes (il s'agit en réalité de proverbes et d'expressions lexicalisées) sont regroupés par chapitres, en référence aux espèces auxquelles ils font allusion, respectivement les poissons (en général), les serpents et anguilles, le šāl (ou zaqzūq, petit poisson du Nil), le tilapia (ou chromis), le silure, le barbeau, le lébis, le mulet salé, le crabe et le dauphin. Pour les oiseaux, l'aigle, le faucon, le milan, le vautour, le corbeau, la bergeronnette grise, la foulque, la grue, le hibou et la chouette, la huppe, le cormoran, le héron, le faux-ibis, le paon, l'autruche, le pigeon et la colombe, l'oie, le canard; les poule, poulet et poulette; le coq, le passereau, les oiseaux en général, enfin l'œuf. On n'en sera pas surpris, le nombre de proverbes par chapitre est très variable, certains n'en contenant qu'un seul. Les modalités qui ont présidé à l'ordre suivi dans la présentation sont expliquées p. 10 et les parties pour lesquelles il n'y a pas « d'ordreparticulier »signalées (ibid.). Une table des matières plus détaillée que celle qui est proposée en arabe et en français, limitée aux deux entrées "poissons » et « oiseaux » aurait été la bienvenue.

Les chapitres suivent tous la même logique. Pour chaque espèce, une petite présentation générale précède les proverbes. Elle peut décrire l'animal 
concerné, évoquer les raisons pour lesquelles il est devenu proverbial ou expliquer la manière dont il est chassé ou pêché. Suite à cela, dans la partie en arabe, chaque proverbe est mentionné, précédé par un numéro. Dans la partie en français, chaque proverbe est présenté à la fois en arabe et dans sa traduction française, avec le même numéro que dans la partie en arabe. Le proverbe est suivi par un bref paragraphe, en arabe ou traduit en français, dans lequel sont généralement précisées sa signification et les conditions de son emploi, parfois des explications lexicales, ou des traits anecdotiques liés à son utilisation ou supposés expliquer le processus de proverbialisation.

Pour déterminer l'apport de l'ouvrage à la littérature populaire égyptienne, il est indispensable de se demander comment peut être estimée "l'égyptianité" des proverbes recueillis. À cet égard, le corpus soulève au moins trois questions : celle du degré de parenté de certains proverbes avec le patrimoine ancien et savant en langue arabe ; celle de l'emploi de certains proverbes dans d'autres régions du monde arabe. La troisième question concerne la diachronie : quelle part de cet intéressant corpus est toujours en usage?

Avant de revenir en détail sur les deux premières questions, quelques mots à propos de la troisième. Comme cela a été dit plus haut, les sources utilisées, rapidement décrites p.4-8, sont des sources écrites qui s'étendent sur plusieurs siècles. Assembler ces proverbes par thème a un intérêt indéniable, mais l'absence de toute différenciation entre les énoncés qui sont toujours dans l'usage, ceux qui l'étaient encore il y a peu et ceux qui ne le sont plus, parfois depuis fort longtemps, aurait apporté un éclairage précieux. Présentés en synchronie (à quelques exceptions près, tel le proverbe 87 qui est daté), ces proverbes, dictons ou expressions risquent d'apparaître à un lecteur non averti comme étant, tous, toujours également vivants dans l'usage. Certes, il est des proverbes qui ont la vie dure et qui traversent aisément les siècles et les régions, pour diverses raisons qu'il n'est pas lieu de développer ici. Mais nombre d'autres sont éphémères de sorte que le mélange adopté dans le recueil, s'il sert l'approche thématique, n'en est pas moins gênant.

Les deux autres questions, mentionnées plus haut, ont trait, l'une et l'autre, à des faits de langue, de culture et d'histoire des textes. Le lecteur ne pourra manquer de se demander ce qu'il faut entendre précisément par l'expression Proverbes égyptiens, figurant dans letitre del'ouvrage. L'introduction laisse entendre qu'il s'agit moins de la prendre dans une acception large que d'insister sur l'existence " d'un caractère proprement égyptien» (p.2) pour ce corpus. C'est pourquoi, sans démentir ce qui a été dit sur le caractère plaisant de l'ouvrage ni contester sa richesse et son utilité pour une meilleure connaissance du patrimoine proverbial populaire ou savant, quelques réserves sont ici de rigueur.

En effet, de nombreux proverbes classés dans l'ouvrage en tant que proverbes égyptiens le sont davantage dans un élan enthousiaste qu'au terme d'une démarche raisonnée d'un point de vue linguistique et/ou anthropologique. Il n'est guère possible de considérer, par exemple, l'énoncé inna al-buġāt bi-ardị-nā yastansir (p. 49/p.38), déjà cité dans les 
Amātī d'al-Qālī (m. 967 ?), les Proverbes d'al-Maydānī (m. 1124) ou les Maqāmāt d'al-Ḥarīīi (m. 1122) (au passage, il s'agit de trois auteurs abbassides "iraquiens ») comme un proverbe "égyptien». On peut faire la même remarque à propos de l'expression gurāb al-bayn (p. 58), déjà présente dans le Kitāb al-'ayn d'al-Hुalīl (m. 786 ?) et dont les attestations poétiques les plus célèbres sont chez les poètes 'udrites hiğaziens. Plus généralement, tous les éléments recueillis, qui peuvent se retrouver dans des sources antérieures à l'époque mamelouke, notamment dans le Kitāb al-ḥayawān d'al-Ǧāhiz (puisqu'il est précisé, p. 8, qu'il est utilisé comme source et référence explicite) auraient dû être présentés comme des énoncés appartenant au patrimoine culturel commun au monde arabo-musulman médiéval savant, plutôt que comme des énoncés égyptiens typiques et vivants. Les proverbes cités par les auteurs mamelouks ou plus tardifs ne peuvent être davantage spécifiquement rattachés à une aire géographique égyptienne, aussi longtemps qu'on les retrouve dans d'autres sources de même époque, composées dans d'autres régions de l'Orient, même ceux que cite $\mathrm{Ibšin}^{\mathrm{i}}{ }^{1}$, qui était "égyptien" et plus sensible que d'autres à l'aspect vernaculaire et populaire de la langue.

Ces proverbes anciens posent donc un problème de classification, à la fois géographique et culturel. Mais ils en posent également un autre sur le plan linguistique. Bien qu'il n'y ait pas de barrière étanche entre langue arabe littérale et langues vernaculaires ou

1. C'est la vocalisation retenue par l'Encyclopédie de l'Islam, pour cet auteur également désigné comme Abīhī (c'est le cas dans notre ouvrage). dialectes, il est un peu délicat de ne pas tenir compte de ces deux espaces linguistiques distincts, surtout quand des siècles les séparent. Les incidences de la diglossie sur les choix faits par l'auteur en ce qui concerne la vocalisation des proverbes ne sont pas sans susciter des interrogations, le système adopté n'étant ni régulier ni explicité. En effet, autant on peut comprendre que les voyelles désinentielles soient remplacées par un sukūn, s'agissant des énoncés en égyptien, langue dans laquelle les déclinaisons sont absentes, autant il est inattendu de voir les énoncés en arabe littéral, tantôt entièrement vocalisés, tantôt partiellement vocalisés (voir par exemple proverbe 324), enfin vocalisés parfois avec les codes de l'arabe dialectal. L'irrégularité se vérifie d'un proverbe à l'autre: dans le proverbe 324 , la voyelle permettant de faire la liaison n'est pas donnée mais elle l'est dans 333. Elle s'observe, parfois, à l'intérieur du même proverbe. Ainsi, dans le proverbe 333, qui vient d'être cité, on peut lire buġātu ț-tayri aktar firāhnan (seul le terme en gras n'a pas de désinence). Cela est troublant pour le lecteur averti, même s'il peut rétablir lui-même les règles syntaxiques ou phonétiques. Mais cela est, de plus, problématique pour le lecteur qui serait moins confirmé en langue arabe.

Une autre modalisation opportune aurait consisté à ne pas perdre de vue que certains des proverbes recueillis sont également en usage dans d'autres régions ou pays du monde arabe. Certes, il est pratiquement impossible de retracer le cheminement de ces proverbes; de même, il est impensable, sans un travail de recherche engageant plusieurs personnes sur une longue durée, de faire 
une collecte exhaustive de la totalité des proverbes en cours dans diverses régions (complétant les travaux qui existent déjà). Pour autant, il est un peu délicat de présumer à ces énoncés une "naissance » forcément égyptienne. Par exemple, à un mot près (zayy est remplacé par $\left.m^{e} t l\right)$, le proverbe 207 "Comme l'oie, maternelle sans mamelles » s'employait encore en Syrie dans les années 1950. Il peut être compris aujourd'hui par la transparence de sa signification, mais il n'est plus dans l'usage. Par contre, on emploie toujours en Syrie et au Liban, « Acheter du poisson dans la mer (bahr)» (proverbe 7) pour " vendre la peau de l'ours avant de l'avoir tué ». Certes, bahr désignera pour les uns la Méditerranée et pour les autres le Nil, mais on voit bien comme il peut être délicat de localiser spécifiquement ces propos. L'ouvrage contient donc de nombreux proverbes utilisés ailleurs qu'en Égypte avec la même signification et dans le même contexte. Il en inclut aussi quelques-uns qui s'emploient ailleurs dans un contexte différent. Ainsi, l'expression rapportée en 337 'alā ț-țāyir (au vol), dont il est dit qu'elle s'emploie pour désigner « celui qui aborde une affaire sans étude ni examen » (p. 151) s'emploie couramment au Liban pour désigner celui qui a un esprit vif et capte les choses rapidement.

Par-delà les défauts qui viennent d'être signalés, il ne faut pas oublier la difficulté qu'il peut y avoir à constituer de tels corpus. Le nombre de proverbes réunis est à lui seul et en tant que tel un indice de la richesse du terrain exploré. Cette collecte, qui fixe un corpus en partie éphémère, est une démarche utile et intéressante, même si elle n'est pas tout à fait académique.

Katia ZAKHARIA

Professeur à l'université Lyon 2/ CNRS

GREMMO- UMR 5195 